Editorial

\title{
Acknowledgment to Reviewers of World Electric Vehicle Journal in 2020
}

\author{
World Electric Vehicle Journal Editorial Office
}

Citation: World Electric Vehicle Journal Editorial Office.

Acknowledgment to Reviewers of World Electric Vehicle Journal in 2020

World Electr. Veh. J. 2021, 12, 40

https://doi.org/10.3390/wevj12010040

Published: 11 March 2021

Publisher's Note: MDPI stays neutral with regard to jurisdictional claims in published maps and institutional affiliations.

Copyright: $\odot 2021$ by the author. Licensee MDPI, Basel, Switzerland.

This article is an open access article distributed under the terms and conditions of the Creative Commons Attribution (CC BY) license (http://creativecommons.org/licenses/by/4.0/).

MDPI AG, St. Alban-Anlage 66, 4052 Basel, Switzerland

Peer review is the driving force of journal development, and reviewers are gatekeepers who ensure that World Electric Vehicle Journal maintains its standards for the high quality of its published papers. Thanks to the cooperation of our reviewers, in 2020, the median time to first decision was 22 days and the median time to publication was 52 days. The editors would like to express their sincere gratitude to the following reviewers for their precious time and dedication, regardless of whether the papers were finally published:

Aarniovuori, Lassi

Abarzadeh, Mostafa

Ahmed, Hafiz

Alfredo, Testa

Ali, Muhammad Umair

Aljohani, Tawfiq

Almadhor, Ahmad

Anvari-Moghaddam, Amjad

Balakhontsev, Alexander

Băldean, Doru-Laurean

Barreras, Jorge

Basbas, Socrates

Bause, Katharina

Beltran-Carbajal, Francisco

Berzi, Lorenzo

Bisaglia, Carlo

Bivona, Enzo

Brendel, Alfred Benedikt

Burgio, Alessandro

Burzyński, Damian

Cao, Mengqiu

Cao, Yuan

Carlos Ferreira, João

Caux, Stéphane

Chang, Che-Jung

Chatzikyriakou, Despoina

Chawla, Yash

Chen, Boli

Chen, Chen

Chen, Fuming

Chen, Guan-Ren

Chen, Kuo-Ching

Chen, Mengyuan
Chindamo, Daniel

Choe, Song-yul

Choi, Hyunhong

Ciupe, Valentin

Clairand, Jean-Michel

Craciunescu, Aurelian

Cristian-Ioan, Leahu

Danielis, Romeo

Darmon, Ruxandra

Deresveaux, Anatole

Domiri Ganji, Davood

Doukas, Dimitrios

Duarte, Jorge

Eberle, Ulrich

Eccarius, Timo

Eremia, Mircea

Fernández-Ramos, José

Ferreira, Joao

Ferreira, Joao Carlos Amaro

Fowler, Michael

Gandoman, Foad

Gao, Yuting

Gao, Zhiming

Garg, Akhil

Ghatak, Kamalika

Giglioli, Romano

Giraldo, Michael

Girovský, Peter

Gong, Xun

González, Manuela

Graham, Robert

Guilbert, Damien

Han, Manbae 
Helber, Stefan

Hernandez, Leobardo

Hiermann, Gerhard

Hinov, Nikolay

Hong, Tianqi

Hossain, Md Alamgir

Hoyos Velasco, Fredy

$\mathrm{Hu}$, Yong

$\mathrm{Hu}$, Zechun

Huang, Wenjing

Huh, Jun-Ho

Iclodean, Calin Doru

Ing, Chye Ing

Ingale, Nilesh

Jeffs, James

Jiao, Xiaohong

Jon, Ander López-Ibarra

Kaewpradap, Amornrat

Kaminski, Marcin

Kandasamy, Nandha Kumar

Kaplon, Andrzej

Karlis, Athanasios

Kelleher, Luke

Khatib, Tamer

Kim, Donghyun

Knap, Vaclav

Kołodziej, Janusz

Koltsaklis, Nikolaos

Kopczyński, Artur

Kowald, Matthias

Kozlov, Andrey

Kühl, Alexander

Laclair, Tim J.

Ladygin, Anatoly N.

Lanusse, Patrick

Lazarou, Stavros

Lebel, Félix-Antoine

Łebkowski, Andrzej

Lee, Kyo-Beum

Lencwe, Mpho

$\mathrm{Li}$, Jie

Li, Liang

Li, Siqi

Lim, Kai Li

Lim, Ocktaeck

Lin, Zhenhong

Liu, Teng

Lopez-Garcia, Irvin

Lu, Fei

Lu, Shaofeng

Luque, Pablo Rodríguez
Macht, Gretchen

Manai, Bilal

Mantovani, Sara

Márquez-Fernández, Francisco J.

Marra, Dario

Massier, Tobias

McGuire, Michael

Mehmood, Khawaja Khalid

Miao, Bingrong

Minh, Ta Cao

Minnaert, Ben

Miranda-Vidales, Homero

Misul, Daniela

Monzon Verona, Jose Miguel

Moradpour, Milad

Możaryn, Jakub

Nahidi, Asal

Nazari, Ashkan

Neubauer, Jeremy

Nezamoddini, Nasim

Nguyen, Bao-Huy

Noussan, Michel

$\mathrm{Ou}$, Shiqi

Ouyang, Dongxu

Panchal, Satyam

Panzavecchia, Nicola

Papanikolaou, Nick

Parra, Alberto

Pedrosa, Delfim

Peng, Ming-Tsan

Pereira, Guillermo Ivan

Philipsen, Ralf

Puskar, Michal

Qi, Cong

Quddus, Md Abdul

Quintero, Carlos

Radac, Mircea-Bogdan

Rahmani, Fatemeh

Ramea, Kalai

Rangarajan, Shriram S.

Rhi, Seok-Ho

Ricciardi, Vincenzo

Rigas, Emmanouil S.

Rinderknecht, Stephan

Ro, Jong-Suk

Ruangjirakit, Kitchanon

Rubino, Sandro

Rubio, Jose De Jesus

Rybarczyk, Dominik

Safari, Momo

Safoutin, Michael J. 
Salazar, Mauro

Savard, Christophe

Scamarcio, Alessandro

Schueppel, Fabian

Scorrano, Mariangela

Serpi, Alessandro

Setiyo, Muji

Shams Ghahfarokhi, Payam

Shao, Yunli

Shen, Sheng

Shiu, Eric C.

Sivaneasan, Bala Krishnan

Song, Wentao

Spanoudakis, Polychronis

Subramanian, Vignesh

Sun, Qun

Sun, Wei

Sun, Weichao

Sun, Xiaodong

Suomalainen, Emilia

Szinai, Julia

Tchagang, Alain

Teijón-López-Zuazo, Evelio

Trimboli, Scott

Triviño, Alicia

Tsai, Mi-Ching

Ustun, Ozgur

Vafaeipour, Majid

Verkroost, Lynn

Vignati, Michele

Villante, Carlo

Wager, Guido

Waldron, Julie

Wang, Shiyuan
Wang, Xiaoya

Watson, Neville

Wittmann, Michael

Wojciechowski, Daniel

Wolbertus, Rick

Woo, Seong Tak

Wu, Jinglai

$\mathrm{Wu}, \mathrm{Yu}$

Xu, Nan

Yahya, Bernardo Nugroho

Yamamoto, Toshiyuki

Yang, Jiaqiang

Yang, Li

Yang, Shichun

Yang, Zhile

Yeboah, Godwin

Yokochi, Alexandre

Yu, Yuanbin

Zahedmanesh, Arian

Zerhouni, Noureddine

Zhang, Anpeng

Zhang, Junzhi

Zhang, Lijun

Zhang, Qi

Zhang, Xizheng

Zhao, Ying

Zhou, Bin

Zhu, Juner

Zhu, Xiaoyuan

Zhu, Ziqiang

Zou, Changfu

Zubizarreta, Asier

Zuo, Wenjie 